\title{
Estudio de biodiscos como tratamiento secundario de aguas residuales domésticas
}

\author{
Rbc study as domestic wastewater secundary treatment
}

\author{
Gustavo Delgado Sandoval ${ }^{*}$ \\ 1 CITRAR, Universidad Nacional de Ingeniería, Av. Túpac Amaru S/N, Puerta N%, Rímac, Perú.
}

Recibido (Recieved): 20/10/2018 Aceptado (Accepted): 10/05/2019

\begin{abstract}
RESUMEN
Se diseñó un sistema de biodiscos a escala de laboratorio como tratamiento secundario de un efluente de un reactor UASB. El sistema se construyó con dos etapas en serie, cada etapa con un volumen efectivo de 81 litros y siete (07) discos de PVC de 60 $\mathrm{cm}$ de diámetro que hacían un área total efectiva de $7.48 \mathrm{~m}^{2}$. El sistema funcionó a un caudal constante de 432 L/día (TRH de 4.5 horas por etapa) y se evaluó en dos períodos de operación variando la velocidad de rotación de los discos ( 3 rpm durante 3 meses y $5 \mathrm{rpm}$ durante 3 meses). Se midieron los valores de Demanda Bioquímica de Oxígeno $\left(\mathrm{DBO}_{5}\right)$, Sólidos Suspendidos Totales (SST), Turbiedad, pH y Temperatura. Además se observaron las características físicas y microbiológicas de la biopelícula durante todo el período de investigación. El sistema tuvo un grado óptimo de oxigenación, alcanzando solo en la primera etapa un valor promedio de $5.21 \mathrm{mg} \mathrm{O}_{2} /$ l. La eficiencia de remoción promedio de la $\mathrm{DBO}_{5}$ fue del $90 \%$ para una velocidad de rotación de $3 \mathrm{rpm}$ y del $78 \%$ para $5 \mathrm{rpm}$. Finalmente el sistema mostró un mejor desempeño en términos de remoción de materia orgánica para una velocidad de $3 \mathrm{rpm}$. Además, se comprobó que dos etapas en serie tuvieron un mejor rendimiento que una sola etapa. Los microorganismos más representativos de la biopelícula de la primera etapa fueron protozoarios ciliados nadadores o libres y las bacterias filamentosas que tuvieron un crecimiento excesivo para el segundo período. En la segunda etapa, fueron los protozoarios ciliados fijos, rotíferos y algas unicelulares y filamentosas.
\end{abstract}

Palabras Clave: Biodiscos, UASB, DBO, Tratamiento, Aguas residuales.

\section{ABSTRACT}

Laboratory model RBC system was designed as secondary treatment of an UASB reactor effluent. RBC System was built with two stages in series, each stage with effective volume of 81 liters and seven (07) PVC disks of $60 \mathrm{~cm}$ diameter with a total effective area of $7.48 \mathrm{~m}^{2}$. RBC system operated at a constant flow rate of $432 \mathrm{I} /$ day (HRT of 4.5 hours) and was operated in two periods at different disk rotational speed ( $3 \mathrm{rpm}$ for 3 months and $5 \mathrm{rpm}$ for 3 months). The values of BOD $_{5}$, TSS, Turbidity, pH and Temperature were measured. Moreover, the physical and microbiological biofilm characteristics were observed throughout the study period. RBC System achieved optimal degree of oxygenation, with an average value of $5.21 \mathrm{mg}$ DO/I just in the first stage. DOB5 Removal efficiency was $90 \%$ for $5 \mathrm{rpm}$ of rotational speed and $78 \%$ for $3 \mathrm{rpm}$. Finally, the RBC system showed a better performance in terms of organic matter when the discs were rotated at $3 \mathrm{rpm}$. Moreover, it was found that two stages performance in series was higher than a single stage. The most representative biofilm microorganisms in first stage were protozoa ciliated swimmers or free and filamentous bacteria that had an excessive growth at second period. In the second stage, it was fixed ciliated protozoa, rotifers and unicellular and filamentous algae.

Key Words: RBC, UASB, DOB, Wastewater, Sewage treatment.

\section{INTRODUCCION}

El tratamiento de aguas residuales domésticas mediante contactores biológicos rotatorios, o también llamados biodiscos, ha sido investigado muy poco en Perú. Debido a las ventajas como su alta eficiencia de remoción de materia orgánica, bajo costo de operación y mantenimiento, cortos tiempos de retención hidráulica y por consecuencia una reducida área requerida y bajo consumo de energía respecto a otros procesos aerobios es de vital importancia realizar investigaciones para poder implementar en las zonas de bajos recursos. Este sistema podría ser implementado como tratamiento primario o como tratamientos secundario, por ejemplo después de un tanque imhoff.

\footnotetext{
* Corresponding author.:

E-mail: gdelgados@uni.pe

DOI: https://doi.org/10.21754/tecnia.v29i1.545
} 
Los biodiscos ( $\mathrm{RBC}$ ) son sistemas de tratamiento biológico aerobio de aguas residuales. En el caso de RBC, la biomasa se presenta simultáneamente en la forma de biopelícula fija (adheridas a los discos) y biopelícula en suspensión (como biomasa en el reactor). Aunque en mayor medida y la de mayor importancia es la biopelícula fija.

Consiste en un medio de soporte formado por discos de algún material en específico. Estos discos pueden estar parcial o totalmente sumergidos en el agua residual, y son montados paralelos entre sí y perpendicular al eje de soporte. El eje de soporte está unido a un motor mecánico que le da una velocidad de rotación a los discos.

Inmediatamente después del arranque del sistema, los microorganismos presentes en el agua residual comienzan a adherirse al medio en movimiento y a desarrollarse.

El rendimiento de los RBCs depende de varios parámetros de diseño. Los de mayor importancia son: Velocidad rotacional de los discos, tasas de carga orgánica e hidráulica, tiempo de retención hidráulico (TRH), medio de soporte, número de etapas, temperatura, $\mathrm{pH}$, características del agua residual y la biopelícula, niveles de oxígeno disuelto (OD), recirculación de lodos y efluente y sumergencia o inmersión de los discos [2].

\section{MATERIALES Y MÉTODOS}

Durante los meses de Junio a Diciembre del año 2018, en la planta de aguas residuales piloto del CITRAR de la UNI, se armó un sistema de biodiscos a escala de laboratorio como tratamiento secundario. La planta piloto cuenta con un sistema de pre-tratamiento compuesto por una cámara de rejas y un desarenador. Seguidamente el agua residual pre-tratada ingresa a un Reactor Anaerobio de Manto de Lodos de Flujo Ascendente (UASB) que tiene un tiempo de retención hidráulico aproximado de ocho horas. Parte de este afluente se derivaron a los biodiscos.

El sistema de biodiscos se diseñó para tratar un caudal constante de 432 l/día y estuvo conformado por dos etapas de iguales medidas conectadas en serie, cada una con un volumen de 100 litros (incluyendo un sedimentador tipo imhoff de 20 litros en el fondo) con TRH total del sistema de 9.0 horas.

El procedimiento del cálculo de la superficie requerida, se basó asumiendo una eficiencia de remoción de materia orgánica del 90\%. Para ello se utilizó el modelo matemático semi-empírico basado en el balance masas y cinética de Monod propuesto por Kornegay (1975) [1].

$$
Q_{0}\left(S_{0}-S_{2}\right)=P A \frac{S_{1}}{K_{S}+S_{1}}+P A \frac{S_{2}}{K_{S}+S_{2}} .
$$

Donde: Qo: Caudal de aplicación (I/día); So: DBO de ingreso (mg/l); $\mathrm{S}_{1}$ : DBO a la salida de la 1era etapa $(\mathrm{mg} / \mathrm{l})$; $\mathrm{S}_{2}$ : DBO a la salida de la 2 da etapa $(\mathrm{mg} / \mathrm{l}) ; \mathrm{K}_{\mathrm{s}}$ y $\mathrm{P}$ : Parámetro experimentales; A: Superficie de contacto total requerida $\left(\mathrm{m}^{2}\right)$.

Como medio de contacto se utilizaron siete (07) discos de PVC por cada etapa que tenían un diámetro de $60 \mathrm{~cm}$ y un espesor promedio de $4 \mathrm{~mm}$ que hacían un total de área efectiva de $7.48 \mathrm{~m}^{2}$ (Ver figura 1 y 2). Los discos fueron lijados con una amoladora para darle mayor rugosidad a la superficie y así mejorar la capacidad de adherencia de la biopelícula. Los discos se montaron en un eje de fierro de $3 / 8$ " que se conectó a un motor graduado con velocidades de $3 \mathrm{rpm}$ y $5 \mathrm{rpm}$. El porcentaje de sumergencia de los discos fue del $40 \%$.

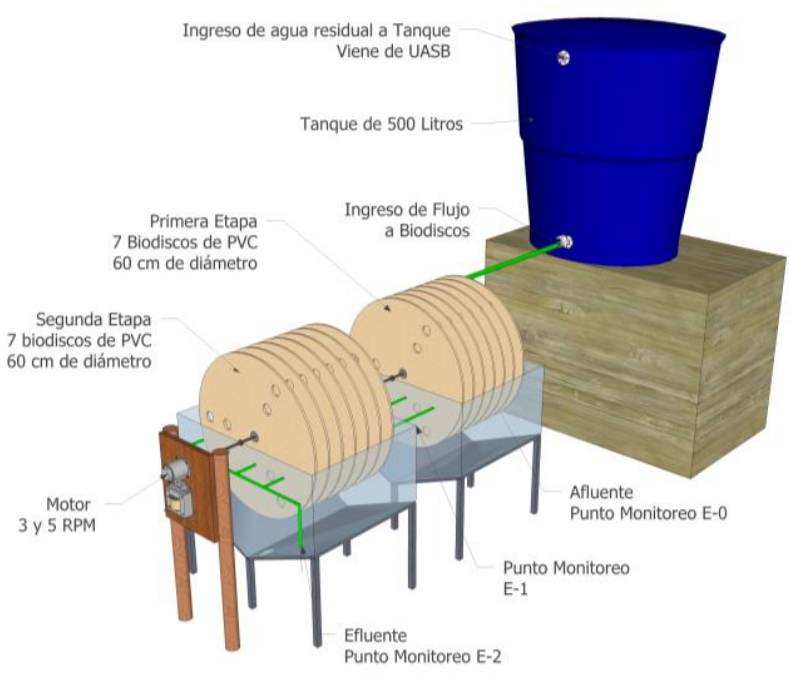

Figura 1. Esquema del sistema de Biodiscos.

El sistema de biodiscos trató un afluente con valores promedios de la $\mathrm{DBO}_{5}$, SST, Turbiedad y OD de: $134.5 \pm 28.8 \mathrm{mg}-\mathrm{DBO}_{5} / \mathrm{l}, 28.0 \pm 7.1 \mathrm{mg} \mathrm{SST} / \mathrm{l}, 51.3 \pm 17.0 \mathrm{UNT}$ y $0.3 \pm 0.3 \mathrm{mg}-\mathrm{O}_{2} / \mathrm{l}$ respectivamente. Durante el desarrollo de la investigación el pH tuvo una variación de 6.19 a 8.72 y la temperatura de $15.9{ }^{\circ} \mathrm{C}$ a $24.9{ }^{\circ} \mathrm{C}$. El estudio se dividió en dos períodos de evaluación con velocidades de rotación de discos diferentes:

- Velocidad de $3 \mathrm{rpm}$ durante 3 meses ( $Q=432 \mathrm{l} / \mathrm{día})$

- Velocidad de $5 \mathrm{rpm}$ durante 3 meses (Q=432 l/día)

Durante este tiempo se midieron la Temperatura, $\mathrm{pH}$, $\mathrm{OD}$, Turbiedad, $\mathrm{DBO}_{5}$ y SST en cada etapa. Para ello se propuso tres puntos de monitoreo (Ver figura 1): E-o (afluente), E-1 (Salida de la primera etapa) y E-2 (Salida de la segunda etapa).

Además se registró con fotografías el desarrollo de la biopelícula durante toda la investigación y se adquirieron micrografías ópticas con el fin de identificar los principales microorganismos que habitan en la biopelícula. 


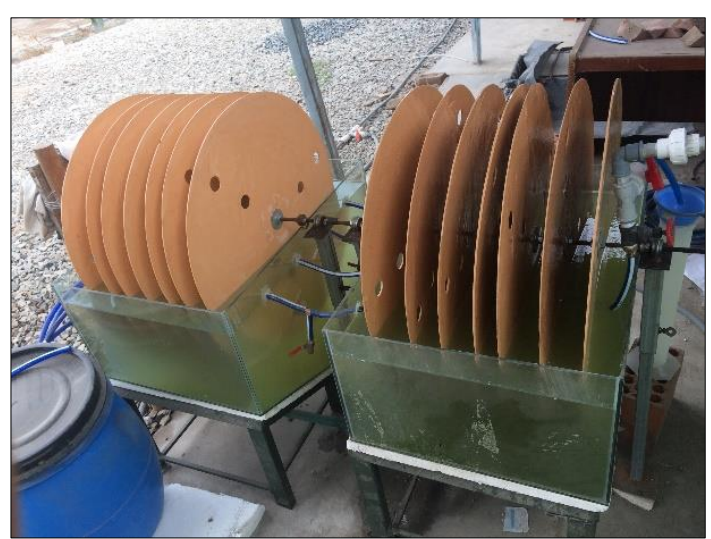

Figura 2. Biodiscos instalados.

\section{DISCUCIÓN DE RESULTADOS}

Las variaciones del OD, $\mathrm{DBO}_{5}$ y los SST del afluente y efluente de cada etapa se muestran en la tabla 1 y en las figuras 7 al 12.

En la primera etapa y en un TRH de 4.5 horas se logró oxigenar la masa de agua proveniente del UASB $(\mathrm{OD}=0.3 \pm 0.3 \mathrm{mg} / \mathrm{l})$, a valores promedio de $4.18 \pm 1.13 \mathrm{mg} / \mathrm{l}$ para una velocidad de rotación de $3 \mathrm{rpm}$ y de 5.21士1.33 para una velocidad de rotación de $5 \mathrm{rpm}$, cantidades de oxígeno suficiente para que se lleve a cabo un proceso aerobio. Este valor se fue incrementando conforme atravesaba las etapas de biodiscos. De la figura 6 , se puede observar valores picos de OD, como en la semana
6, donde se alcanzó valores de $8.06 \mathrm{mg} / \mathrm{l}$ que puede ser producto del superávit de oxígeno producido por las algas y la ausencia de microorganismos debido al primer desprendimiento de la biopelícula. De la Figura 8, podemos ver que cuando se aumentó la velocidad de rotación, el valor promedio de OD se incrementó. Este incremento de $O D$ en el agua fue favorable para que nuevos microorganismos, como los rotíferos, poblaran la biopelícula de la 2da etapa, que se identificaron durante el periodo de evaluación con $5 \mathrm{rpm}$. Investigadores como Friedman et al 1979, encontraron que la rapidez de utilización del sustrato aumenta con la velocidad de rotación, ya que velocidades altas mejoran la trasferencia de oxígeno [9].

Como podemos ver en la figura 10, la velocidad de rotación de los discos provocó un cambio significativo en los valores promedios de la DBO5 para ambas etapas de biodiscos. Realizando un análisis estadístico de ANOVA ( se comprueba la hipótesis planteada para esta investigación que la velocidad de rotación de los discos influyó en la remoción de la $\mathrm{DBO}_{5}$. A mayor velocidad de rotación, la eficiencia del sistema decrece. Una de las razones de que sucediera esto es que a mayor velocidad de rotación de los discos, la adherencia de la biopelícula a estos se hace más difícil, además se pone en suspensión los lodos depositados en el fondo de los reactores. Esto se corroboró con el aumento de los valores de turbiedad y de sólidos suspendidos.

Tabla 1. Valores Promedios de monitoreo

\begin{tabular}{llll|lll}
\hline & \multicolumn{5}{c}{ Biodiscos } \\
\cline { 2 - 7 } & $\begin{array}{l}\text { Afluente } \\
(\mathrm{E}-\mathrm{O})\end{array}$ & $\begin{array}{l}\text { 1era Etapa } \\
(\mathrm{E}-1)\end{array}$ & $\begin{array}{l}\text { 2da Etapa } \\
(\mathrm{E}-2)\end{array}$ & $\begin{array}{l}\text { Afluente } \\
(\mathrm{E}-\mathrm{0})\end{array}$ & $\begin{array}{l}\text { 1era Etapa } \\
(\mathrm{E}-1)\end{array}$ & $\begin{array}{l}\text { 2da Etapa } \\
(\mathrm{E}-2)\end{array}$ \\
\hline $\mathrm{DBO}_{5}(\mathrm{mg} / \mathrm{l})$ & 130.53 & 27.32 & 13.82 & 127.11 & 52.50 & 27.62 \\
$\mathrm{SST}(\mathrm{mg} / \mathrm{l})$ & 26.18 & 16.40 & 13.23 & 24.73 & 20.65 & 12.35 \\
Turbiedad (UNT) & 47.87 & 46.21 & 17.28 & 52.79 & 58.29 & 16.99 \\
$\mathrm{OD}(\mathrm{mg} / \mathrm{l})$ & 0.43 & 4.18 & 7.10 & 0.24 & 5.21 & 7.66 \\
$\mathrm{PH}$ & 7.32 & 7.66 & 7.83 & 7.24 & 7.63 & 7.59 \\
\hline
\end{tabular}

De acuerdo a la Figura 11, existen dos valores atípicos de SST en los efluentes de la primera y segunda etapa que son muy elevados, con un valor mayor al afluente. Estos valores picos coinciden con las fechas de desprendimientos masivos de la biopelícula que al caer en forma de biosólidos elevan los valores de sólidos suspendidos. De la figura 12, la investigación muestra que el incremento de la velocidad de rotación de los discos de $3 \mathrm{rpm}$ a $5 \mathrm{rpm}$, produce un aumento de los valores de SST en la primera etapa. Esto debido a que una mayor velocidad periférica en los discos provoca un ligero desprendimiento de la biopelícula, otra de las razones es que una mayor agitación y turbulencia pone en suspensión los lodos sedimentados en el fondo del reactor. Durante el período de evaluación de $5 \mathrm{rpm}$ hubo una mayor presencia de bacterias filamentosas, lo cual aumenta los valores de SST.

La biopelícula presentó características propias en cada etapa, teniendo colores gris y marrón en la primera etapa y un color pardo y verde en la segunda etapa (Ver figura 3). Visualmente se pudo observar que la biopelícula de la primera etapa era de mayor espesor que la segunda etapa. 


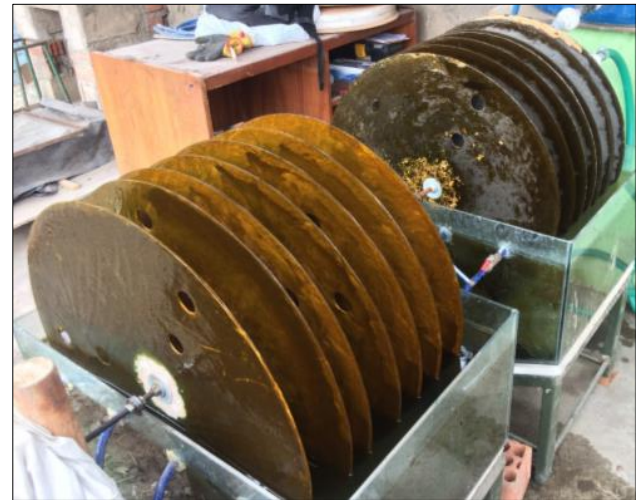

Figura 3. Biopelícula formada en discos de PVC.

Las características físicas y microbiológicas de la biopelícula fueron influenciadas por el aumento en la velocidad de rotación de los discos. Notándose una biopelícula más uniforme y estable con una velocidad de 3 rpm. Un aumento de velocidad originó pérdidas de biopelícula (en mayor medida en la 1era etapa) y por lo tanto disminuyó el área efectiva bajando el rendimiento del sistema.

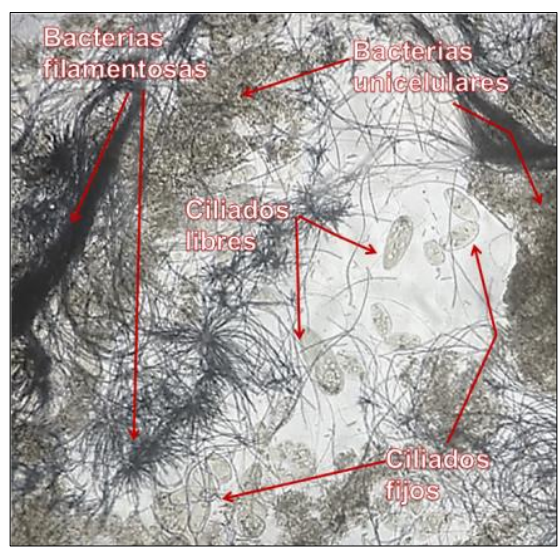

Figura 4. Principales microorganismos (1era etapa)

Las poblaciones identificadas en la biopelícula variaron de acuerdo a la etapa en que se encontraban. Predominando, principalmente, en la primera etapa las bacterias unicelulares, bacterias filamentosas y ciliados libres o nadadores (Ver figura 4 y 5). Se observaron bacterias filamentosas que tenían un aspecto velloso de color blanco y conforme fue avanzando la investigación, fueron poblando más áreas de los discos. Debido a que el afluente proviene de un reactor anaerobio donde la concentración del oxígeno disuelto es cero y con concentraciones de sulfuro de hidrógeno $\left(\mathrm{H}_{2} \mathrm{~S}\right)$, es probable que las bacterias filamentosas observadas en esta etapa sean microorganismos de la especie beggiatoa, thiothrix, leptothrix y/o sphaerotilus natans, que según la bibliografía y diversos autores evidencian su presencia en sistemas de biodiscos [3-8].

En la segunda etapa predominaron las algas verdes unicelulares, diatomeas y ciliados fijos. Otros protozoos que se observó en la biopelícula fueron del género amebas. Los nemátodos también se identificaron en mayor número que en la primera etapa, de diversos tamaños y tipos. El aumento de velocidad influyó en la aparición de nuevos microorganismos en la segunda etapa, como los rotíferos (Ver figura 6). Esto puede explicarse por el aumento de los niveles de oxígeno disuelto (con un promedio de $7.66 \mathrm{mg} / \mathrm{l}$ ) a causa del incremento de la velocidad de rotación de los discos. Los rotíferos fueron favorables en la remoción de sólidos suspendidos y de bacterias unicelulares. Lo cual se demostró con los valores de SST y turbiedad.

\section{CONCLUSIONES}

-Los contactores biológicos rotatorios (biodiscos) construidos según los parámetros de diseño mencionados tuvieron una eficiencia alta en la remoción de la materia orgánica como tratamiento secundario de un efluente de un UASB con un caudal de 432 I/día y una $\mathrm{DBO}_{5}$ promedio de $134.5 \mathrm{mg} / \mathrm{l}$. Para una velocidad de rotación de discos de $3 \mathrm{rpm}$, se logró una eficiencia de remoción de $\mathrm{DBO}_{5}$ del $90 \% \pm 3 \%$. Mientras que para una velocidad de $5 \mathrm{rpm}$, se obtuvo una eficiencia del $78 \% \pm 7 \%$. De esta manera se comprobó que aumentos de velocidad de rotación influyen de forma negativa a la eficiencia del sistema. El sistema de biodiscos logró una máxima eficiencia de remoción de materia orgánica en términos de $\mathrm{DBO}_{5}$ del $93 \%$ con un valor en el efluente de $7.90 \mathrm{mg} / \mathrm{l}$.

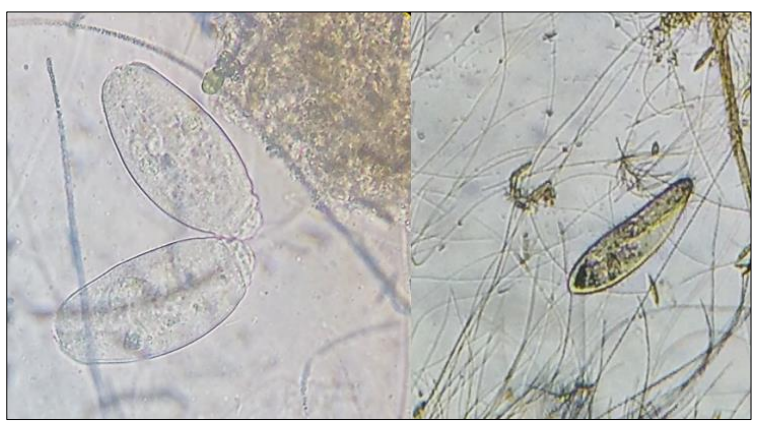

Figura 5. Ciliados encontrados en la 1era etapa. Izquierda: Ciliado Fijo; Derecha: Ciliado Libre.

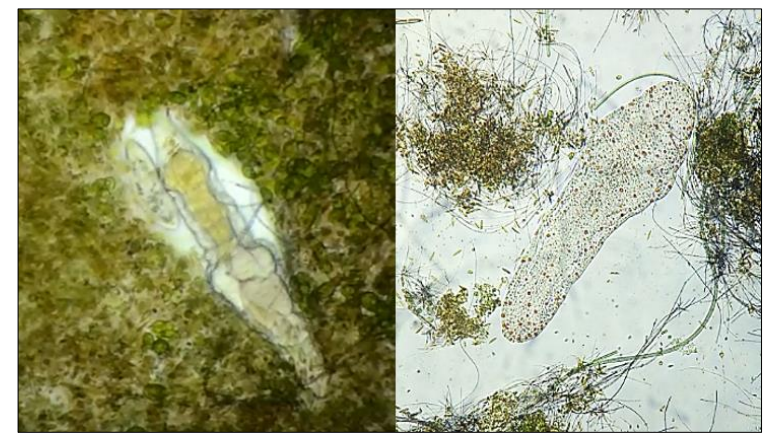

Figura 6. Rotíferos observados en la 2da etapa 
-La eficiencia de remoción de materia orgánica en términos de $\mathrm{DBO}_{5}$, turbiedad y sólidos suspendidos totales, muestran que dos etapas de biodiscos conectadas en serie tuvieron una mayor remoción que solo una etapa.

-El aumento de la velocidad de rotación de los discos de $3 \mathrm{rpm}$ a $5 \mathrm{rpm}$, incrementó los valores de oxígeno disuelto en el agua de $4.18 \mathrm{mg} / \mathrm{l}$ a $5.21 \mathrm{mg} / \mathrm{l}$ para la primera etapa y de 7.10 a $7.66 \mathrm{mg} / \mathrm{l}$ para la segunda etapa. Lo cual demuestra que con la velocidad de rotación se puede controlar los niveles de oxígeno disuelto en los reactores.

-La primera etapa fue la que removió la mayor parte de la $\mathrm{DBO}_{5}$, llegando alcanzar una eficiencia máxima de remoción del $82 \%$ y una eficiencia promedio del $79 \% \pm 3 \%$ para una velocidad de $3 \mathrm{rpm}$. Un incremento de velocidad de rotación de los discos a $5 \mathrm{rpm}$ tuvo un efecto negativo en la eficiencia de remoción de la primera etapa, bajando su eficiencia promedio al $58 \% \pm 5 \%$.

-Los valores de turbiedad en la primera etapa fueron influenciados en su mayoría por los desprendimientos de la biopelícula adheridas a los discos, además de las bacterias filamentosas que también generaban turbiedad al agua. En la primera etapa no se removió turbiedad, por el contrario aumentó ligeramente cuando la velocidad de los discos se aumentó a $5 \mathrm{rpm}$. Por el contrario, en la segunda etapa si hubo remoción de turbiedad con una eficiencia total del sistema del

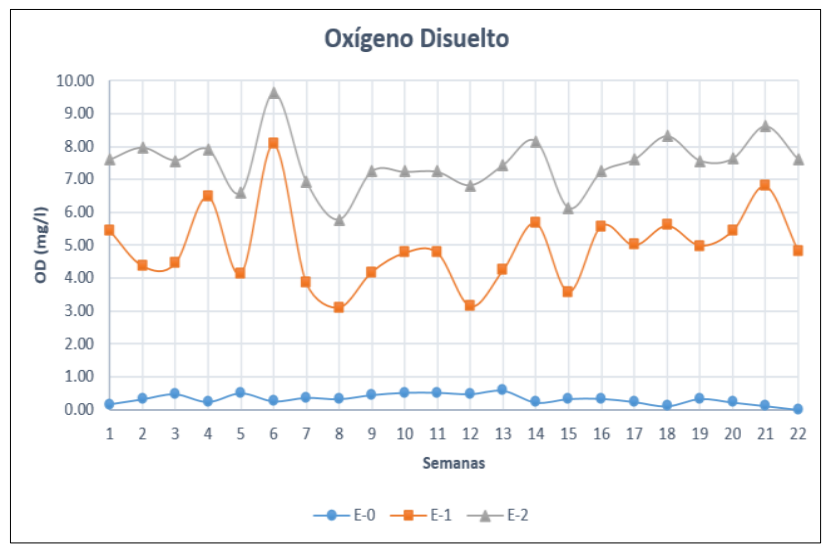

Figura 7. Variación del OD en el tiempo
$63.90 \%$. Se comprobó que el aumento de la velocidad de rotación de los discos de $3 \mathrm{rpm}$ a $5 \mathrm{rpm}$ tuvo una ligera influencia en la primera etapa, mientras que en la segunda etapa no hubo influencia.

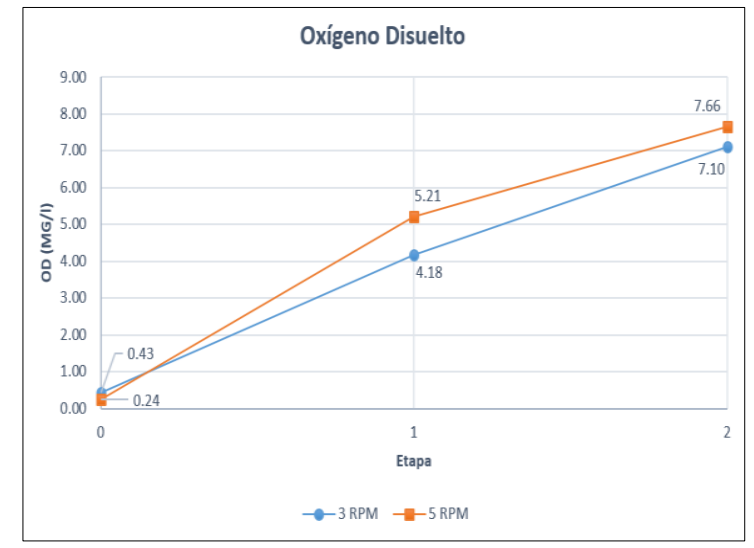

Figura 8. OD por etapas con la velocidad de rotación. 


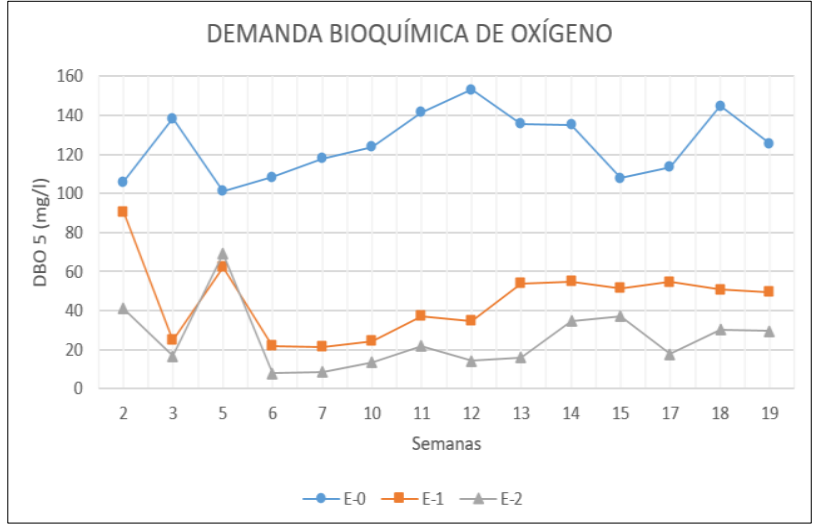

Figura 9. Variación de la $\mathrm{DBO}_{5}$ en el tiempo

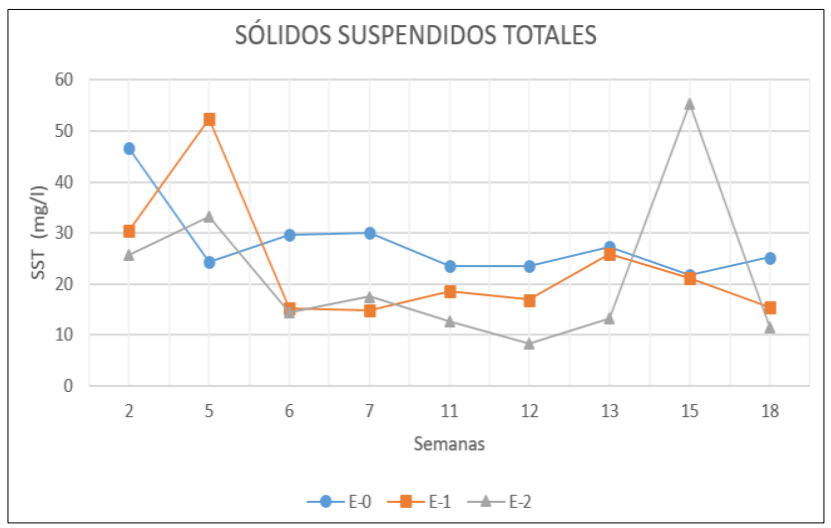

Figura 11. Variación de los SST en el tiempo

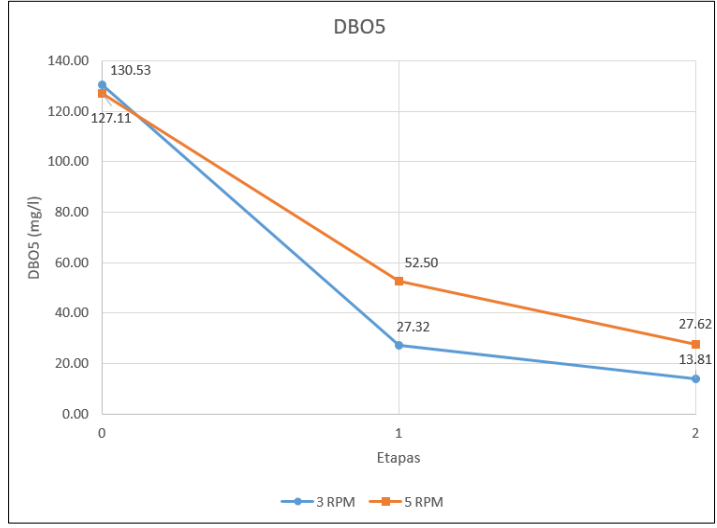

Figura 10. $\mathrm{DBO}_{5}$ por etapas con la velocidad de rotación.

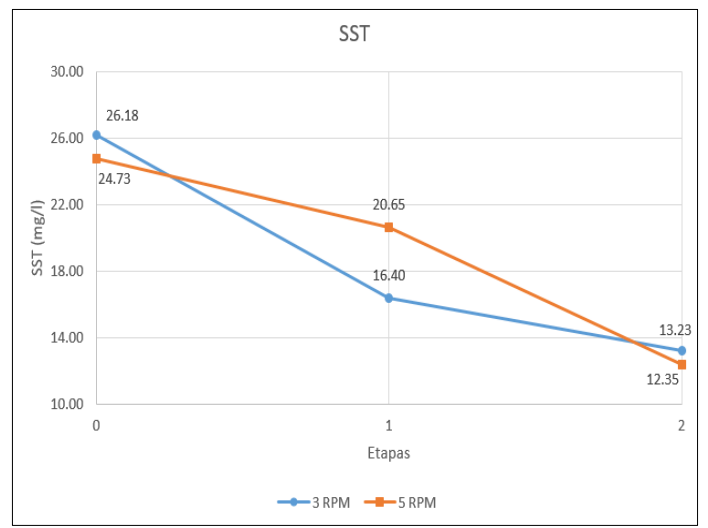

Figura 12. SST por etapas con la velocidad de rotación.
-El sistema de biodiscos tuvo una eficiencia de remoción de SST del $51 \% \pm 9 \%$ para una velocidad de 3 $\mathrm{rpm}$. El aumento en la velocidad de rotación de los discos a $5 \mathrm{rpm}$ no tuvo un impacto significativo en la remoción de SST con un valor de eficiencia del $53 \% \pm 2 \%$. $\mathrm{Si}$ bien es cierto que el tratamiento por biodiscos no tiene como función la remoción de sólidos, se logró remover una fracción por mecanismos de sedimentación, adsorción y la absorción en la biopelícula.

-Se corroboró que una de las desventajas de los biodiscos son las fallas mecánicas del motor y del eje por rotura. Durante la investigación se tuvo muchas fallas del eje por fractura del eje y sobrecalentamiento del motor más aún cuando se incrementó la velocidad de rotación de los discos. Es de vital importancia realizar un diseño detallado del eje considerando el peso de los discos, el peso de la biopelícula, la velocidad de giro y la corrosión por contacto con las aguas residuales.

-Se observó que la eficiencia del sistema disminuye durante la caída de la biopelícula inactiva produciendo sólidos flotantes en el efluente que disminuyen la calidad del agua. Por ello es necesario implementar una unidad de clarificación o sedimentador posterior al sistema.

\section{AGRADECIMIENTOS}

Quisiera dar un profundo agradecimiento a la phD. Rosa Yaya Beas por brindarme su apoyo y la oportunidad de desarrollar esta investigación en las instalaciones del CITRAR. Al Ing. Otto Rosasco Gerkes, profesor investigador de la FIA-UNI, por asesorarme durante la investigación y a todo el personal del Laboratorio $\mathrm{N}^{\circ} 20$ de la Facultad de Ingeniería Ambiental, donde se realizaron los análisis de laboratorio. 


\section{REFERENCIAS}

[1] Ramalho R S 1993 Tratamiento de aguas residuales, Quebec, Canadá. (Ed. Reverte)

[2] Cortez S et al 2008 Rotating biological contactors: a review on main factors affecting performance. Environment Science Biotechnology 7 pp 155-172

[3] Romero José María et al s/f. La biopelícula en los procesos RBC. Facultad de Ingeniería. Universidad Católica de Córdoba: Colombia

[4] Stenstrom M. K. 1981 Use of the rotating biological contactor for appropriate technology wastewater treatment. Graduate Research Engineer. School of Engineering and Applied Science. University of California: L.A

[5] Martín Cereceda M. et al 2001 Confocal and light microscope examination of protozoa and other microorganisms in the biofilms from a rotating biological contactor wastewater treatment plant. Acta Protozoológica 40 pp 263-272

[6] Jae-Chun Chung and Peter F. Strom 1991 Microbiological study of ten New Jersey rotating biological contactor wastewater treatment plans. Research Journal WPCF, Volume 63, Number 1, pp 35-43

[7] Singh V, Mittal A K 2012 Characterization of biofilm of a rotating biological contactor treating synthetic wastewater. IWA publishing Water Science \& Technology 66

[8] Pérez-Uz B, et al. 1998 Biofilm characterization of several wastewater tratment plants with rotating biological contactors in Madrid (Spain). Water Science \& Technological, vol 37, No 4-5, pp 215-218

[9] Friedman A, et al. 1978 Effect of disk rotational speed on biological contactor efficiency. Department of Civil Engineering. Tennesee Technological University, Cooksville, Tenn

cC) $\Theta$ Los artículos publicados por TECNIA pueden ser compartidos a través de la licencia Creative Commons: CC BY-NC-ND 2.5 Perú. Permisos lejos de este alcance pueden ser consultados a través del correo revistas@uni.edu.pe 